\title{
Estimating Surface Normals from Spherical Stokes Reflectance Fields
}

\author{
Giuseppe Claudio Guarnera ${ }^{1, \star}$, Pieter Peers ${ }^{2}$, \\ Paul Debevec ${ }^{1}$, and Abhijeet Ghosh ${ }^{1}$ \\ 1 USC Institute for Creative Technologies, Playa Vista, CA, USA \\ 2 The College of William \& Mary, Williamsburg, VA, USA
}

\begin{abstract}
In this paper we introduce a novel technique for estimating surface normals from the four Stokes polarization parameters of specularly reflected light under a single spherical incident lighting condition that is either unpolarized or circularly polarized. We illustrate the practicality of our technique by estimating surface normals under uncontrolled outdoor illumination from just four observations from a fixed viewpoint.
\end{abstract}

\section{Introduction}

Accurate shape and appearance estimation is a crucial component in many computer vision applications. Despite being at the focal point of intense research, shape and appearance estimation remains a challenging problem, especially under uncontrolled real-world conditions. Estimation of both small-scale surface shape as well as surface reflectance greatly benefits from accurate knowledge of surface orientation. In this work, we propose a novel method for estimating surface orientation from the Stokes polarization vector under a single spherical incident illumination condition that is either circularly polarized (Fig. 1, c-d) or unpolarized (Fig. 1, e-f).

Polarization cues have previously been employed to separate diffuse and specular reflectance components (e.g., [1-4]), to classify materials (e.g., [1, 5]), to estimate reflectance properties (e.g., [6, 7]), and to estimate surface normals (e.g., [8-12]). Motivated by the polarization characteristics of natural lighting, which is either unpolarized (overcast sky) or linearly polarized (sunlight), most of these methods, with exception of [2, 4, 10] focus solely on linear polarization cues. In contrast, in this work we leverage observations of the view-independent symmetric Stokes reflectance field - which encodes the impact of unpolarized, linearly polarized, as well as circularly polarized reflected light - for estimating surface normals under constant incident spherical illumination. We show that for many dielectric materials, a non-negligible circularly polarized reflectance is observed, not only under circularly polarized incident lighting, but also for unpolarized lighting. Leveraging these circular polarization cues greatly improves normal estimation for front facing surfaces. We demonstrate that both types of incident lighting can be used to reliably estimate surface normals from observations of the Stokes reflectance field, and show how this theory can be applied to normal estimation under uncontrolled outdoor illumination.

* Currently at the Department of Mathematics and Computer Science, University of Catania, Italy.

A. Fusiello et al. (Eds.): ECCV 2012 Ws/Demos, Part II, LNCS 7584, pp. 340-349, 2012.

(c) Springer-Verlag Berlin Heidelberg 2012 

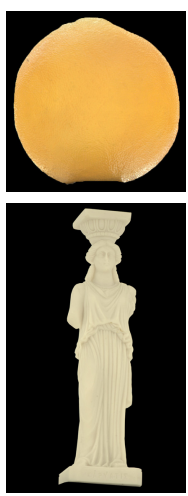

(a) Subject
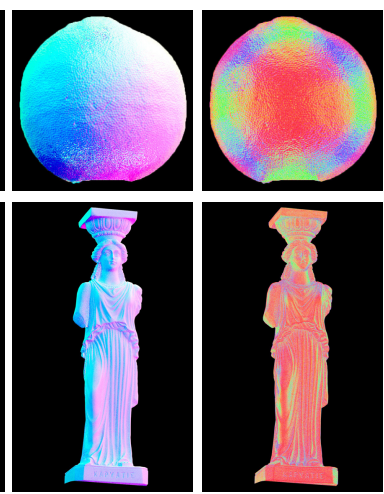

(b) Photometric Normals (c) Circular Stokes Param
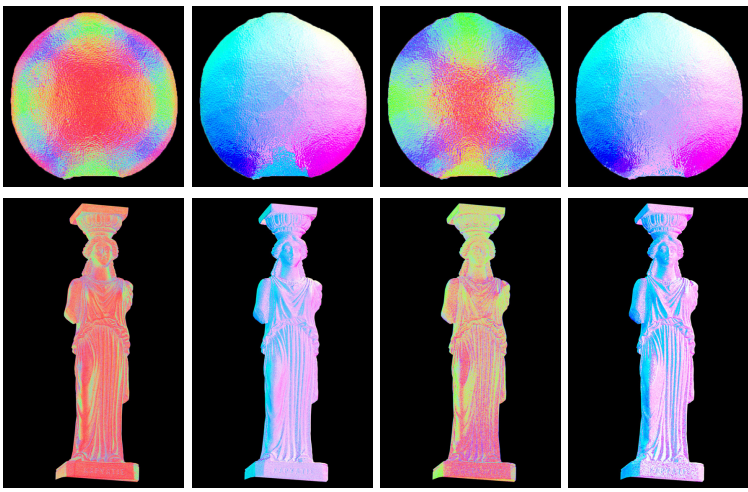

(d) Circ. Pol. Normals

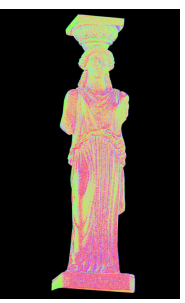

(e) Unpol. Stokes Param.

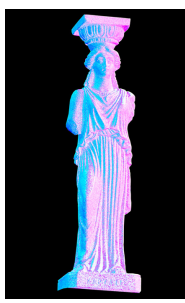

(f) Unpol. Normals

Fig. 1. Estimating surface normals (encoded as $\frac{1}{2}(\mathbf{x}+1) \rightarrow R, \frac{1}{2}(\mathbf{y}+1) \rightarrow G$, and $\frac{1}{2}(\mathbf{z}+1) \rightarrow B$ ) from Stokes parameters (encoded as $\left|s_{3}\right| \rightarrow R,\left|s_{1}\right| \rightarrow G$, and $\left|s_{2}\right| \rightarrow B$ ) of specularly reflected incident spherical illumination. Surface normals inferred from circularly polarized illumination (c-d), and unpolarized illumination (e-f), compared to surface normals obtained from a photometric stereo variant [2](b). Top-row: Plastic orange - $\phi$-ambiguity resolved by growing normals inward. Bottom-row: Marble statue - $\phi$-ambiguity resolved using an additional measurement.

\section{Related Work}

The seminal work by Woodham [13] on photometric stereo, which proposes to estimate surface normals from single viewpoint images of diffuse surfaces lit from different lighting directions, has inspired much research since its introduction in 1978. While recent advances do not require the incident lighting to be known beforehand [14], these methods still require multiple lighting conditions to accurately estimate surface orientation. In contrast the proposed method requires just a single lighting condition, making it better suited for normal estimation in uncontrolled outdoor environments.

Shape estimation under fixed lighting can be obtained from specular cues. Roth and Black [15] introduce the concept of specular flow and illustrate how such specular flow relates to shape under general environmental illumination for surfaces that exhibit (binary) mixtures of diffuse and specular materials. Vasilyev et al. [16] extend specular flow to general unconstrained surface shapes. However, estimating specular flow from multiple viewpoints is a difficult and complex problem. While the proposed method also relies on specular reflections, it only needs four observations from a single viewpoint under constant illumination, avoiding complex specular flow and/or correspondence computations. Furthermore the proposed method naturally handles per-pixel mixtures of diffuse and specular.

Polarization cues have also been used extensively for estimating surface normals. Most methods exploit the property that the angle of polarization relates to the surface normal direction perpendicular to the plane of incidence. The direction within the plane of incidence is found by either observing the surface from multiple viewpoints [17-20], or from the degree of polarization [11, 12, 21-23]. The majority of the above methods infer surface information from a series of photographs of the surface while rotating a 
linear polarizer in front of the camera. The proposed method does not only rely on linear polarization cues, but instead infers surface information from the full characterization of the reflectance (i.e., the Stokes reflectance field), that can be captured in just four photographs with different polarizers in front of the camera (i.e., three linear polarizers rotated 0, 45, and 90 degrees, and a (left) circular polarizer). Furthermore, we show that even under unpolarized incident lighting, some non-negligible circularly polarized reflectance is present, which impacts the accuracy of the estimated normals if ignored.

Ma et al. [2] propose a photometric stereo variant that employs either linear or circular polarization for separating diffuse and specular reflections, and compute accurate surface normals from the specular component. However, unlike the proposed method, they rely on photometric cues for estimating the surface normal.

Koshikawa [10] estimate the surface normal of a single surface point from the Stokes vector, measured using a ellipsometer, under a single directional circularly polarized light source. They assume known index of refraction, and estimate the normal from the Fresnel equations. However, they ignore the ambiguity in the azimuthal angle of the surface normal. In contrast, the proposed method employs a standard DSLR camera instead of an ellipsometer and considers both circularly polarized and unpolarized incident lighting, and explicitely handles the azimuthal ambiguity.

\section{Surface Normal Estimation from Stokes Vectors}

Background: Mueller Calculus. Before detailing how surface normals can be estimated from measurements of the Stokes reflectance field under a single spherical illumination condition, we first give a brief overview of the related theory on Stokes parameters and Mueller calculus. A more detailed description can be found in [24]. In what follows we assume that the surface consists of a homogeneous dielectric material, and the coordinate frame is that of the camera, i.e., the camera looks down the $-\mathrm{Z}$ axis, the $\mathrm{X}$ axis points right, and the $\mathrm{Y}$ axis points up.

The polarization state of light can be described by the 4-element Stokes vector $\mathbf{s}=$ $\left(s_{0}, s_{1}, s_{2}, s_{3}\right)$, where: $s_{0}$ represents the total power, $s_{1}$ is the power of the $0^{\circ}$ linear polarization, $s_{2}$ is the power the $+45^{\circ}$ linear polarization, and $s_{3}$ is the power of right circular polarization. The degree of polarization, or ratio of the power of polarized light versus the total power equals: $D O P=\sqrt{s_{1}^{2}+s_{2}^{2}+s_{3}^{2}} / s_{0}$. When a polarized ray hits a reflective surface (i.e., specular), the resulting change to Stokes vector is predicted by Mueller calculus: $\mathbf{s}^{\prime}=\mathbf{C}(\phi) \mathbf{D}(\delta ; \mathbf{n}) \mathbf{R}(\theta ; \mathbf{n}) \mathbf{C}(-\phi) \mathbf{s}$. Each of the linear operators $\mathbf{C}, \mathbf{D}$ and $\mathbf{R}$ can be compactly represented by a matrix. $\mathbf{C}$ is the Mueller rotation that brings the linear polarization parameters into a canonical frame:

$$
\mathbf{C}=\left(\begin{array}{cccc}
1 & 0 & 0 & 0 \\
0 & \cos 2 \phi & -\sin 2 \phi & 0 \\
0 & \sin 2 \phi & \cos 2 \phi & 0 \\
0 & 0 & 0 & 1
\end{array}\right)
$$

where $\phi$ is the angle between the camera's $\mathbf{x}$ axis and the incident plane determined by the surface normal $\mathbf{n}$ and incident direction (i.e., $\arccos (\mathbf{n} \cdot \mathbf{x})$ ). $\mathbf{R}$ is the Mueller reflection matrix: 


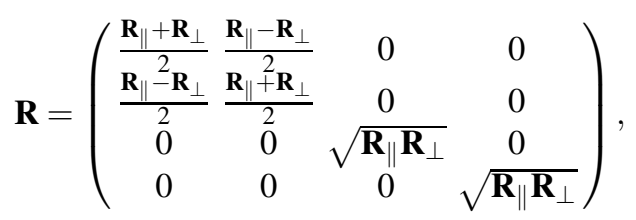

where $\mathbf{R}_{\|}$and $\mathbf{R}_{\perp}$ are the Fresnel equations for the parallel and perpendicular components, respectively, as functions of the incident angle $\theta$ (i.e., $\arccos (\mathbf{n} \cdot \mathbf{z})$ ). Finally, $\mathbf{D}$ is the Mueller retardation matrix. For dielectric materials, this essentially flips the signs of $s_{2}$ and $s_{3}$ over the Brewster angle.

Finally, the Stokes reflectance field [6] is defined as the description of the Stokes vectors resulting from a single surface interaction, under a user-defined incident light field, computed for every surface normal direction.

Uniform Circularly Polarized Incident Lighting. We will develop our theory first on the case where the incident illumination has power $\Phi$ over the sphere of incident directions and is circularly polarized. In other words $\mathbf{s}(\omega)=\mathbf{s} \sim(\Phi, 0,0, \Phi)$. Applying Mueller calculus for diffuse and specular surface interactions yields the Stokes reflectance vector $\mathbf{s}^{\prime}$ :

$$
\begin{aligned}
\mathbf{s}^{\prime} & =\left(s_{0}^{\prime}, s_{1}^{\prime}, s_{2}^{\prime}, s_{3}^{\prime}\right) \\
& =\left(\rho_{s} \Phi \frac{\mathbf{R}_{\perp}+\mathbf{R}_{\|}}{2}+\rho_{d} \Phi, \rho_{s} \Phi \frac{\mathbf{R}_{\perp}-\mathbf{R}_{\|}}{2} \cos 2 \phi, \rho_{s} \Phi \frac{\mathbf{R}_{\perp}-\mathbf{R}_{\|}}{2} \sin 2 \phi, \mp \rho_{s} \Phi \sqrt{\mathbf{R}_{\|} \mathbf{R}_{\perp}}\right),
\end{aligned}
$$

where $\rho_{d}$ and $\rho_{s}$ are the diffuse and specular albedo of the dielectric material. Note that $s_{0}^{\prime}$ is the result of both specular as well as diffuse reflectance. While the impact of diffuse reflectance can be easily removed from $s_{0}^{\prime}$ using the degree of polarization [6], we avoid using this component in our computations to minimize the impact of potential diffuse pollution (e.g., due to suboptimal polarizers). Note that $s_{1}^{\prime}, s_{2}^{\prime}$ and $s_{3}^{\prime}$ are the result of specular reflections only, and hence unaffected by any diffuse pollution.

To compute $\theta$, we establish a relation $\chi$ between the Stokes components $s_{1}^{\prime}, s_{2}^{\prime}$ and $s_{3}^{\prime}$ that is independent of specular albedo $\rho_{s}$ and the power of the incident light source $\Phi:$

$$
\chi=\arctan \left(\frac{s_{3}^{\prime}}{\sqrt{s_{1}^{\prime 2}+s_{2}^{\prime 2}}}\right)=\arctan \left(\mp 2 \frac{\sqrt{\mathbf{R}_{\|} \mathbf{R}_{\perp}}}{\mathbf{R}_{\perp}-\mathbf{R}_{\|}}\right) .
$$

Note that $\chi$ is implicitly related by a non-linear one-to-one mapping to $\theta$ via the Fresnel equations $\mathbf{R}_{\perp}$ and $\mathbf{R}_{\|}$. We invert this non-linear mapping by precomputing a lookup table that maps $\chi$ to $\theta$, obtained by evaluating Eq. (4) for a dense sample of $\theta$, and assuming a fixed index of refraction of 1.4. Fig. 2 1 illustrates the impact of on the accuracy of the recovered $\theta$ when the true index of refraction differs from the fixed index. As can be seen, the error remains below $5^{\circ}$ for moderate indexes of refraction and for $\theta<60$. Alternatively, the index of refraction can also be computed using the method described in [6]. 


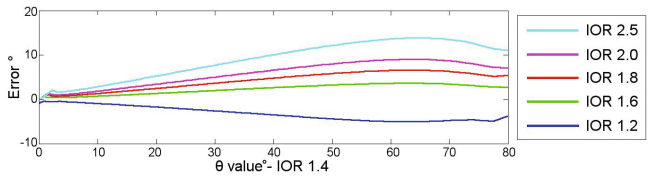

Fig. 2. Error plots for $\theta$ estimated with a fixed index of refraction of 1.4 for materials with increasing index of refraction

The remaining azimuthal angle $\phi$ can be directly computed from the linear components $s_{1}^{\prime}$ and $s_{2}^{\prime}$ :

$$
\arctan \left(\frac{s_{2}^{\prime}}{s_{1}^{\prime}}\right)=\arctan \left(\frac{\sin 2 \phi}{\cos 2 \phi}\right)=2 \phi .
$$

Note that this is exactly the same as the angle of polarization used in prior work. Similarly as in prior work, this relation is ambiguous: $\phi$ and $\phi+\pi$ both satisfy the above equation. Resolving this ambiguity as been the focus of many prior work. In this work, we employ two alternative strategies:

1. For convex objects, we can grow the normals in from the silhouette, assuming that the normals at the silhouette are orthogonal to silhouette edge and the view direction. This strategy was also employed in prior work such as [21].

2. Alternatively, we can capture an additional photograph of the surface while lit by another known spherical illumination condition $I(\cdot, \cdot)$ such that $I(\phi, \theta) \neq I(\phi+$ $\pi, \theta)$. This strategy is useful when incident lighting can be precisely controlled (e.g., laboratory setting). We will also employ a variant of this for normal estimation in uncontrolled outdoor lighting.

Uniform Unpolarized Incident Lighting. In the case that the incident lighting is unpolarized (i.e., the Stokes vector is $(\Phi, 0,0,0)$ ), Mueller calculus for diffuse and specular surface interactions predicts the following resulting reflected Stokes vector:

$$
\mathbf{s}^{\prime}=\left(\rho_{s} \Phi \frac{\mathbf{R}_{\perp}+\mathbf{R}_{\|}}{2}+\rho_{d} \Phi, \rho_{s} \Phi \frac{\mathbf{R}_{\perp}-\mathbf{R}_{\|}}{2} \cos 2 \phi, \rho_{s} \Phi \frac{\mathbf{R}_{\perp}-\mathbf{R}_{\|}}{2} \sin 2 \phi, 0\right) .
$$

Observe that $s_{1}^{\prime}$ and $s_{2}^{\prime}$ are solely due to specular reflections. $\phi$ can be computed similarly as before using Eq. (5). However, Eq. (4) cannot be employed for estimating $\theta$, because the circular Stokes component $s_{3}^{\prime}$ differs. While a relation can be expressed in terms of $s_{1}^{\prime}$ and $s_{2}^{\prime}$ (e.g., $\sim s_{1}^{\prime 2}+s_{2}^{\prime 2}$ ), such a relation will suffer from a low SNR when $\mathbf{R}_{\perp} \approx \mathbf{R}_{\|}$. While no circular polarization is predicted by Eq. (6), we experimentally detected a small quantity of left circularly polarized reflectance under unpolarized incident illumination. We observe that, while fairly constant for different angles of $\theta$, it is stronger than the observed amount of linearly polarized reflectance near normal incidence (Fig. 3, (a)) . This provides a means to improve the accuracy of surface normals for front facing surfaces. We believe that this observed circularly polarized reflectance is due to polarization preserving (subsurface) scattering, which was not taken into account when computing Eq. (6). Fig. 3, (b-e) shows an experimental validation that indicates that the observed circularly polarized reflectance is not due to specular reflections (i.e., non-zero $s_{3}^{\prime}$ is observed between the sharp highlights). Furthermore, it 


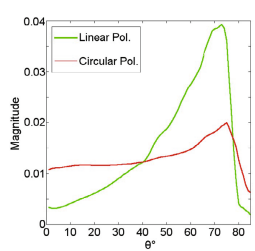

(a) Circular vs Linear mag.

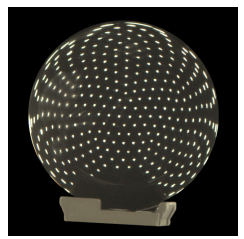

(b) Black ball

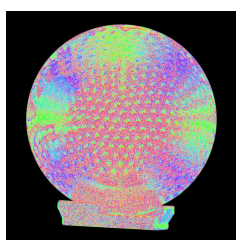

(c) Black ball Unpol. Stokes

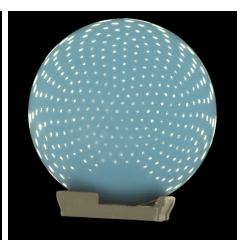

(d) Blue ball

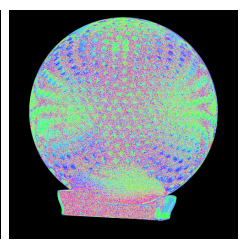

(e) Blue ball Unpol. Stokes

Fig. 3. (a) Plot of reflected circular vs linear polarization under uniform spherical illumination as function of $\theta$. (b - e) Stokes parameters of two sharp specular balls under uniform spherical illumination (emitted from a LED sphere with 346 lights) showing circular polarization (red) between the observed specular highlights which are linearly polarized.

has been shown that scattering from randomly oriented particles can give rise to circular polarization (see [25], p.451), and that polarization is preserved for an average of 2.5 scattering events [26]. Exact modeling of the corresponding Mueller matrix, and thus the resulting $s_{3}^{\prime}$, for polarization preserving scattering is difficult due to the many unknown factors. Instead of relying on an exact formulation of $s_{3}^{\prime}$ for computing $\theta$, an example-based strategy is employed. The Stokes reflectance field of a dielectric object with known shape (e.g., a sphere) is recorded under unpolarized incident lighting. To account for differences in specular albedo and scattering properties, a first-order correction is performed by scaling the maximum of $\sqrt{s_{1}^{\prime 2}+s_{2}^{\prime 2}}$ and of $s_{3}^{\prime}$ in the target dataset to match those of the exemplar dataset. This first-order correction works well for objects that exhibit a rich variation in surface normals. Finally, the normal of a surface point is computed by finding the best matching $\sqrt{s_{1}^{\prime 2}+s_{2}^{\prime 2}}$ and $s_{3}^{\prime}$ pair on the exemplar.

Uncontrolled Outdoor Illumination. The extension of the proposed method to uncontrolled outdoor environments builds on two observations:

- We observe that overcast sky is unpolarized, and the content varies approximately as: $I(\phi, \theta) \sim \sin (\phi)$. Such an illumination condition is slowly varying, and fulfills the condition that $I(\phi, \theta) \neq I(\phi+\pi, \theta)$, and thus it is suitable for resolving the $\phi$-ambiguity.

- Furthermore, if the content of the environment lighting varies slowly in comparison to the sharpness of the specular reflection (which is the case for overcast sky), then we can approximate the intensity of incident lighting over the solid angle of significant specular response as a constant scale factor $s_{\omega}$. Hence, for every surface point, the specular response is similar (up to a scale factor $s_{\omega}$ ) as if it was captured under a constant lighting condition. However, care has to be taken when performing the first-order correction of $\sqrt{s_{1}^{\prime 2}+s_{2}^{\prime 2}}$ and $s_{3}^{\prime}$ since different surface points' specular reflections are possibly scaled by a different scale factor $s_{\omega}$. We propose to either capture the exemplar in the same environment (and hence it includes $s_{\omega}$ ), or alternatively perform a first-order correction on the ratio $s_{3}^{\prime} / \sqrt{s_{1}^{\prime 2}+s_{2}^{\prime 2}}$ instead of the individual components (effectively dividing out $s_{\omega}$ ). The latter is similar to the ratio used in Eq. (4). 
Again, we can readily apply the theory outlined for the uniform unpolarized incident lighting case to compute surface normals under uncontrolled overcast illumination.

The inclusion of circular polarization yields a more robust estimation of surface normals compared to prior work such as [11, 12, 21-23] which rely solely on linear polarization cues to estimate the in-plane incident angle from the reduced degree of polarization: $D O P^{\prime}=\sqrt{s_{1}^{2}+s_{2}^{2}} / s_{0}$ (i.e., $s_{3}$ is implicitly assumed to be zero). We found the estimation error to reduce by $5^{\circ}$ close to normal incidence with the inclusion of circular polarization.

\section{Results}

We validate our theory in both controlled and uncontrolled lighting conditions. We follow the acquisition scheme of [6] to capture the Stokes reflectance field. Specifically, four photographs of a surface are recorded with four different polarizers in front of the camera: a linear polarizer rotated $0^{\circ}\left(P_{H}\right), 45^{\circ}\left(P_{45}\right), 90^{\circ}\left(P_{V}\right)$, and a (left) circular polarizer $\left(P_{\circ}\right)$. We can then robustly compute the Stokes vector components as: $s_{0}=P_{H}+P_{V}$, $s_{1}=P_{H}-P_{V}, s_{2}=2 P_{45}-s_{0}$, and $s_{3}=s_{0}-2 P_{\circ}$. This scheme has the advantage that any unpolarized light that passes through the polarizers will be canceled out in the computation of $s_{1}, s_{2}$, and $s_{3}$. While $s_{0}$ may overestimate the unpolarized power, it is not used in any of our computations.

Fig. 1 shows surface normal estimation results for a convex plastic orange (top row) under controlled incident lighting generated by an LED sphere with 346 lights. We compare the estimated surface normals under circularly polarized and unpolarized spherically uniform incident lighting to surface normals obtained by the photometric method of Ma et al. [2]. The $\phi$ ambiguity is handled by growing the normals in from the silhouette. Fig.1 (bottom row) presents the results under similar conditions of a more complex marble statue with concavities. The $\phi$-ambiguity is solved by capturing an additional lighting conditions: $I(\phi, \theta)=(\sin (\phi)+1) / 2$.

Fig. 4 gives a quantitative error analysis of the surface normal estimation for an object with known shape (i.e., sphere). As can be seen the quality of the estimated normals is good, except close towards extreme angles due to reflection occlusion. Furthermore, the surface normals estimated under circular incident lighting exhibit a better SNR for front facing surfaces compared to those acquired under unpolarized incident lighting. The mean angular error is around $7^{\circ}$ for incident angles less than $75^{\circ}$. Note that normals towards the bottom of the sphere exhibit a larger error due to reflection occlusion from the stand supporting the ball.

Fig. 5 shows normal estimation results under a spherical linear intensity gradient in the top-down direction: $I(\phi, \theta)=(\sin (\phi)+1) / 2$, which simulates an idealized outdoor overcast condition. Fig. 5 (top row) show the estimated normals of a plastic maquette under this simulated lighting condition. The $\phi$-ambiguity was handled by growing in the normals from the silhouette. Fig. 5 center and bottom row, show a marble statue and a plaster bas relief respectively captured under the same single simulated lighting condition. For these two cases, the $\phi$-ambiguity was solved using the intensity information of the incident lighting condition. 

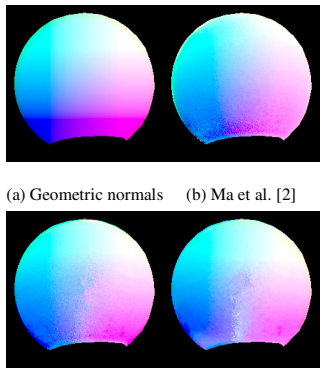

(c) Circ. Pol. Normals (d) Unpol. Normals

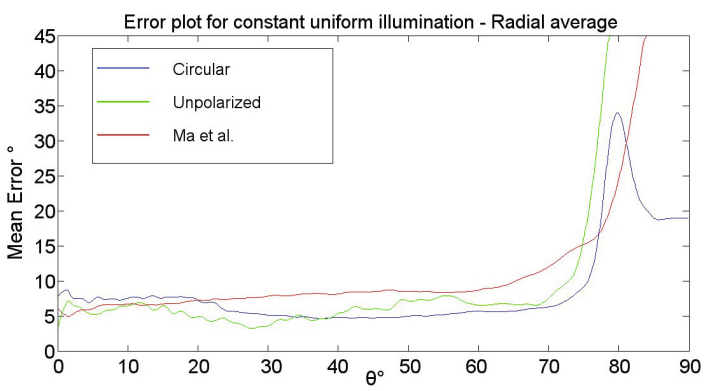

(e) $1 \mathrm{D}$ error plot

Fig. 4. Surface normals of a spherical ball estimated from Stokes parameters of incident spherical illumination. Surface normals inferred from circularly polarized illumination (c), and unpolarized illumination (d), compared to known ground truth geometric normals (a), and compared to normals obtained from linearly polarized incident lighting using the method of [2] (b).
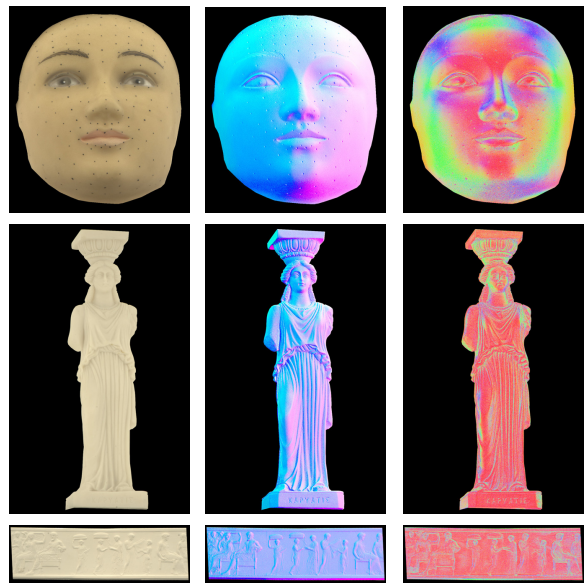

(a) Subject
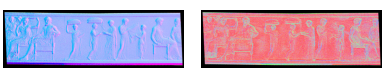

(b) Photometric Normals 2] (c) Circular Stokes Param
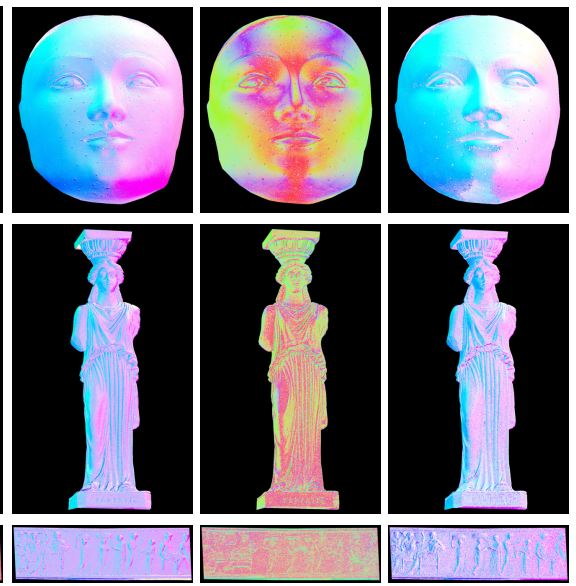

(e) Unpol. Stokes Param.

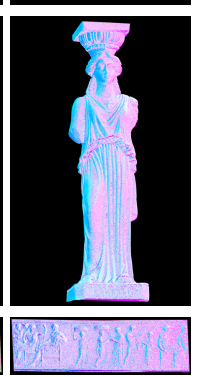

(f) Unpol. Normals

Fig. 5. Estimated surface normals from Stokes parameters under idealized simulated outdoor lighting conditions. Surface normals inferred from circularly polarized illumination (c-d), and unpolarized illumination (e-f), compared to photometric normals [2] (b). Top-row: Plastic maquette - surface normal map of face grown inward from the silhouette. Center-row: Marble statue - $\phi$ ambiguity resolved using directional cues from the incident illumination. Bottow-row: Plaster bas relief.

Fig. 6 shows results of surface normal estimation from outdoor illumination on a cloudy day for the convex plastic orange as well as a jade Confucius statue with several concavities. The exemplar sphere was captured under the same lighting condition. 

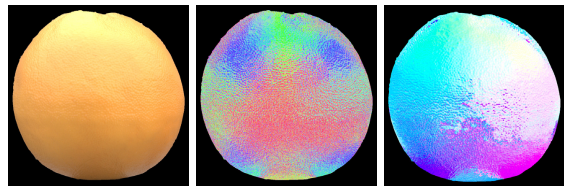

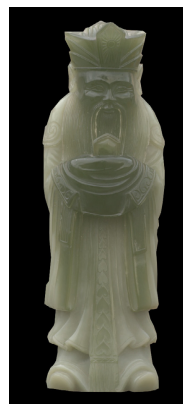

(a) Subject

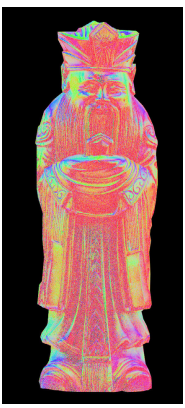

(b) Unpol. Stokes Param

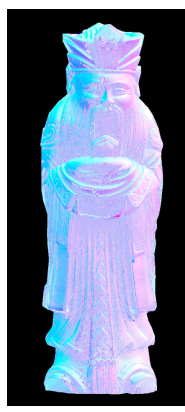

(c) Unpol. Normals

Fig. 6. Estimated surface normals from Stokes parameters of diffuse outdoor illumination. Toprow: Plastic orange. Bottom-row: Confucius statue.

\section{Conclusion}

We presented a novel technique for estimating surface normals from polarization cues obtained from the Stokes reflectance field captured in just four photographs from a single viewpoint and under a single (unpolarized or cicularly polarized) constant spherical incident lighting. While the theory is based on Meuller calculus and is strictly for homogeneous dielectric materials with a constant specular roughness, we found it to work well in practice for both dielectrics as well as dielectric-metal composites. We validated the proposed technique in both controlled as well as uncontrolled outdoor lighting conditions.

Acknowledgments. We would like to thank Jay Busch, Santa Datta, Saskia Mordijck, Kathleen Haase, Bill Swartout, Randy Hill, and Randolph Hall for their support and assistance with this work. This work was sponsored in part by NSF grants IIS-1016703 and ISS-1217765, the University of Southern California Office of the Provost and the U.S. Army Research, Development, and Engineering Command (RDECOM). The content of the information does not necessarily reflect the position or the policy of the US Government, and no official endorsement should be inferred.

\section{References}

1. Wolff, L.B., Boult, T.E.: Constraining object features using a polarization reflectance model. PAMI 13, 635-657 (1991)

2. Ma, W.C., Hawkins, T., Peers, P., Chabert, C.F., Weiss, M., Debevec, P.: Rapid acquisition of specular and diffuse normal maps from polarized spherical gradient illumination. In: Rendering Techniques, pp. 183-194 (2007) 
3. Nayar, S., Fang, X., Boult, T.: Removal of Specularities using Color and Polarization. In: CVPR (1993)

4. Ghosh, A., Fyffe, G., Tunwattanapong, B., Busch, J., Yu, X., Debevec, P.: Multiview face capture using polarized spherical gradient illumination. In: Proceedings of the 2011 SIGGRAPH Asia Conference, SA 2011, pp. 129:1-129:10. ACM, New York (2011)

5. Tominaga, S., Yamamoto, T.: Metal-dielectric object classification by polarization degree map. In: CVPR, pp. 1-4 (2008)

6. Ghosh, A., Chen, T., Peers, P., Wilson, C.A., Debevec, P.: Circularly polarized spherical illumination reflectometry. ACM Trans. Graph. 29, 162:1-162:12 (2010)

7. Cula, O.G., Dana, K.J., Pai, D.K., Wang, D.: Polarization multiplexing and demultiplexing for appearance-based modeling. PAMI 29, 362-367 (2007)

8. Wolff, L.B., Lundberg, A., Tang, R.: Image understanding from thermal emission polarization. In: CVPR, p. 625. IEEE Computer Society, Washington, DC (1998)

9. Miyazaki, D., Ikeuchi, K.: Inverse polarization raytracing: Estimating surface shapes of transparent objects. In: CVPR, pp. 910-917 (2005)

10. Koshikawa, K.: A polarimetric approach to shape understanding of glossy objects, 190-192 (1992)

11. Atkinson, G.A., Hancock, E.R.: Shape estimation using polarization and shading from two views. PAMI 29, 2001-2017 (2007)

12. Saito, M., Sato, Y., Ikeuchi, K., Kashiwagi, H.: Measurement of surface orientations of transparent objects by use of polarization in highlight. J. Opt. Soc. Am. A 16, 2286-2293 (1999)

13. Woodham, R.J.: Photometric stereo: A reflectance map technique for determining surface orientation from image intensity. In: Proc. SPIE's 22nd Annual Technical Symposium, vol. 155 (1978)

14. Basri, R., Jacobs, D.W.: Photometric stereo with general, unknown lighting. In: CVPR, pp. 374-381 (2001)

15. Roth, S., Black, M.J.: Specular flow and the recovery of surface structure. In: Proceedings of the 2006 IEEE Computer Society Conference on Computer Vision and Pattern Recognition, CVPR 2006, vol. 2, pp. 1869-1876 (2006)

16. Vasilyev, Y., Zickler, T., Gortler, S., Ben-Shahar, O.: Shape from specular flow: Is one flow enough?, pp. 2561-2568 (2011)

17. Rahmann, S., Canterakis, N.: Reconstruction of specular surfaces using polarization imaging. In: CVRP, vol. 1, p. 149 (2001)

18. Atkinson, G.A., Hancock, E.R.: Multi-view surface reconstruction using polarization. In: ICCV, pp. 309-316 (2005)

19. Miyazaki, D., Kagesawa, M., Ikeuchi, K.: Transparent surface modeling from a pair of polarization images. PAMI 26, 73-82 (2004)

20. Wolff, L.B.: Surface orientation from two camera stereo with polarizers. In: Proc. SPIE Conf. Optics, Illumination and Image Sensing for Machine Vision IV, vol. 1194, pp. 287-297 (1989)

21. Miyazaki, D., Kagesawa, M., Ikeuchi, K.: Polarization-based transparent surface modeling from two views. In: ICCV, p. 1381 (2003)

22. Atkinson, G.A., Hancock, E.R.: Two-dimensional brdf estimation from polarisation. Comput. Vis. Image Underst. 111, 126-141 (2008)

23. Miyazaki, D., Ikeuchi, K.: Shape estimation of transparent objects by using inverse polarization ray tracing. PAMI 29, 2018-2030 (2007)

24. Collett, E.: Field Guide to Polarization, SPIE Field Guides, vol. FG05. SPIE (2005)

25. Bohren, C., Huffman, D.R.: Absorption and Scattering of Light by Small Particles. Wiley Science Paperback Series (1998)

26. Tuchin, V.V.: Light scattering study of tissues. Physics-Uspekhi 40, 495 (1997) 